\title{
Deterioro cognitivo leve y depresión en el adulto mayor
}

\author{
Ámbar R. Pérez ${ }^{1,2, *}$, Diana C. Oviedo ${ }^{3 *}$, Gabrielle B. Britton ${ }^{4,5 *}$ \\ ${ }^{1}$ Escuela de Psicología, Universidad Católica Santa María la Antigua 0819-08550. \\ ${ }^{2}$ Centro de Neurociencias y Unidad de Investigación Clínica, Instituto de Investigaciones Científicas \\ y Servicios de Alta Tecnología (INDICASAT AIP) 0843-01103, Pmá 5. \\ ${ }^{3}$ Escuela de Psicología, Universidad Católica Santa María la Antigua 0819-08550. \\ ${ }^{4}$ Escuela de Psicología, Universidad Católica Santa María la Antigua 0819-08550. \\ ${ }^{5}$ Centro de Neurociencias y Unidad de Investigación Clínica, Instituto de Investigaciones Científicas \\ y Servicios de Alta Tecnología (INDICASAT AIP) 0843-01103, Pmá 5.
}

*Autor para correspondencia. Email: arperla23@gmail.com, d.ovi@hotmail.com gbritton@indicasat.org.pa

Recibido: 20 de julio de 2018

Aceptado: 03 de agosto de 2018

\section{Resumen}

La depresión y el deterioro cognitivo leve son condiciones comunes en la adultez mayor. Ambos son factores que reducen la independencia del adulto mayor para realizar las actividades de la vida diaria. Estas condiciones son también factores de riesgo para el desarrollo de demencias, en especial la demencia de tipo Alzheimer. Además, aumentan el riesgo de mortalidad cuando se presentan con otras enfermedades crónicas. En este artículo de revisión realizamos una breve descripción de la relación entre depresión y el deterioro cognitivo, la importancia del estudio de ambas, las principales hipótesis que surgen durante el estudio de su comorbilidad y por último los métodos utilizados para su evaluación y diagnóstico.

Palabras claves: envejecimiento, salud mental, cerebro, cognición, neuropsicología.

\footnotetext{
Abstract

Depression and mild cognitive impairment are common conditions in elderly adults. Both are factors that reduce the independence of older adults to perform the activities of daily living. These conditions are also risk factors for the development of dementias, especially Alzheimer's dementia. In addition, they increase the risk of mortality when they co-occur with other chronic diseases. In this review article we describe briefly aspects of the relationship between depression and cognitive impairment, the importance of conducting research focused on these conditions, the main hypotheses that arise during the study of their comorbidity, and lastly the methods employed for their evaluation and diagnosis.
} 
Invest. pens. crit. (ISSN 1812-3864)

Vol. 6, No. 2, mayo - agosto 2018

pp. $05-12$

\section{Introducción}

A nivel mundial, existe un aumento acelerado de la población de adultos mayores de 60 años. En un informe realizado por las Naciones Unidas (1) se estipuló que la población de adultos mayores crecería de $11 \%$ en el 2014 a un $21 \%$ en el 2050. Los adultos mayores de 60 años representan el grupo poblacional de mayor incremento en el mundo, especialmente en regiones que han tenido un rápido crecimiento de desarrollo en las últimas tres décadas, como la de América Latina y el Caribe. Estos cambios demográficos representan una carga significativa los sistemas de salud y la economía de los países.

En Panamá el aumento de la población de adultos mayores de 60 años de edad es consistente con las tendencias a nivel mundial. Entre los años 1960 y 1970 la proporción de adultos mayores en la población se mantuvo estable con un 5.6\% y 5.7\% respectivamente. En el año 2010 se reportó un aumento a $9.7 \%$ de la población, y se proyecta que para el 2050 llegue a 24\% de la población (2). En 1960, las mujeres tenían una expectativa de vida de 62 años que incrementó en el 2015 a 81 años, mientras que en hombres en 1960 la expectativa de vida era de 60 años e incrementó a 75 años en el 2015 (3). El incremento en la expectativa de vida es un reflejo de avances médicos, pero trae consigo otras consecuencias. Una mayor longevidad está relacionada con una mayor cantidad de enfermedades crónicas y morbilidad, lo que a su vez aumenta la discapacidad en los adultos mayores.

Dos condiciones estrechamente asociadas a la edad son la demencia y la depresión (4). La demencia más común, la enfermedad de Alzheimer (EA), representa el 60-80\% de los casos (5). Individuos con deterioro cognitivo leve (DCL) tienen mayor riesgo de padecer EA y deterioro funcional, pero con la coocurrencia de depresión y DCL el riesgo de EA y mortalidad incrementan comparado a individuos sin depresión (6). La depresión es un trastorno del ánimo con síntomas como humor deprimido, perdida de interés o placeres en las actividades de la vida diaria, pérdida o aumento de peso, cambio en los patrones de sueño y el apetito, entre otros (7). Durante la adultez mayor, la depresión esta asociada a factores biológicos y psicosociales (8) y a una peor prognosis (9). Por ejemplo, la depresión se asocia con deterioro en la calidad de vida y mayor riesgo de padecer enfermedades crónicas (10). Conjuntamente la evidencia apunta a que la depresión exacerba múltiples condiciones que perjudican la salud de adultos mayores.

\section{¿Cuáles son los factores sociales y biológicos asociados a la depresión en la vejez?}

Múltiples factores de riesgo psicosociales están implicados en el desarrollo de la depresión en la vejez. El de tipo sociodemográfico más significativo es bajo ingreso económico, que está relacionado con menor acceso a servicios de salud y menor educación (11). Otros factores involucran rasgos de personalidad como apego inseguro, problemas de adaptación, sentimientos de soledad y falta de manejo de sí mismo (12)(13). La falta de apoyo social, reclusión social y declive en las relaciones sociales como la ausencia de amigos o confidentes, son factores causales de depresión y en casos graves incrementan el riesgo de suicidio (14). Incidentes en la vida que causan depresión como la muerte de la pareja o el diagnóstico de una enfermedad crónica también son factores de riesgo (8)(15). 
Invest. pens. crit. (ISSN 1812-3864)

Vol. 6, No. 2, mayo - agosto 2018

pp. $05-12$

La hipótesis de depresión vascular postula que los síntomas depresivos surgen por la comorbilidad entre enfermedades vasculares, factores de riesgo vasculares y lesiones isquémicas(16). Por otro lado, se ha observado que la depresión y la demencia tipo EA comparten comorbilidades médicas, factores de riesgo sociales y biológicos (17)(18). Por ejemplo, individuos con depresión que expresan el gen Apolipoproteina E (APOE-e4), el mayor factor de riesgo genético de la EA esporádica, tienen un mayor riesgo de EA que aquellos sin depresión (19). La EA y la depresión también comparten cambios estructurales del cerebro. Por ejemplo, la desregulación y reducción del hipocampo, en especial en circuitos como el hipocampo-corteza prefrontal, están altamente implicadas en la depresión y EA, y conllevan a déficits cognitivos (18). También la desregulación en el eje hipotalámico-hipofisario-adrenal que resulta en altos niveles de cortisol está asociada a la atrofia hipocampal y pérdida neuronal, características cerebrales de la depresión y EA (20).

\section{¿Existe relación entre la depresión y el DCL?}

La depresión y el DCL son factores de vulnerabilidad en adultos mayores y pueden ocurrir de forma independiente, pero múltiples estudios sugieren que comúnmente la aparición de una está ligada con la otra, y que la presencia de ambas patologías aumenta el riesgo de mortalidad (6). Diversas investigaciones señalan que a menudo coexisten la depresión y DCL. En el estudio poblacional de Envejecimiento Longitudinal Italiano (21), se observó que la prevalencia de depresión en pacientes con DCL era de 63.3\%. De este porcentaje el 49.3\% poseían síntomas de depresión leve y 14.0\% tenía depresión severa. En Latinoamérica, el estudio poblacional del área metropolitana de México encontró dos veces más (52\%) personas con depresión y deterioro cognitivo leve relativo a personas con solo DCL (25.0\%) (11). En Panamá, en un estudio de pacientes ambulatorios, 18.1\% de los participantes presentaban solo depresión, $21.4 \%$ solo DCL y un $11.2 \%$ presentaba depresión y DCL (22). En los tres estudios descritos, se observó más coocurrencia de depresión y deterioro cognitivo en mujeres y en edades más avanzadas, y en personas con mayor cantidad de enfermedades crónicas y limitaciones en las actividades de la vida diaria.

Diferentes hipótesis se han planteado para describir la etiología de la relación entre depresión y DCL. Una hipótesis plantea que las personas con depresión desarrollan DCL en el transcurso del trastorno emocional está sustentada en estudios dónde los tratamientos exitosos para la depresión son congruentes con la mejora en las funciones cognitivas. En un estudio (23) el 55\% de adultos mayores diagnosticados con depresión mayor fueron diagnosticados con DCL, y esta cifra se redujo a $44.8 \%$ posterior a un año de tratamiento para la depresión. Otra hipótesis consiste en que existe una condición neuropatológica en la cual el desarrollo de depresión en la vida adulta refleja un inminente declive cognitivo, y que la presencia de síntomas depresivos podría ser la señal temprana de una enfermedad neurodegenerativa (6). Por último, también se ha planteado que los individuos con algún grado de deterioro cognitivo desarrollan depresión como reacción psicológica ante la presencia de un declive cognitivo y ante la perdida de su independencia para realizar las actividades de la vida diaria (24).

Los factores de riesgo que comparten el DCL y la depresión proporcionan evidencia adicional sobre la etiología de ambas condiciones. En México un estudio (11) reportó que los factores de riesgo más comunes en depresión y DCL son ser mujer, mayor de 75 años de edad, soltero, retirado o jubilado, pensionado y con baja escolaridad. En Panamá los factores de riesgo demográficos fueron similares; 
Invest. pens. crit. (ISSN 1812-3864)

Vol. 6, No. 2, mayo - agosto 2018

pp. $05-12$

los participantes de mayor edad (mayores de 80 años) y con menor educación mostraron mayor probabilidad de coocurrencia. Específicamente, participantes con cuatro o más condiciones crónicas y con limitaciones en la actividades de la vida diaria estaban más predispuestas a padecer ambas condiciones (22). También se han encontrado asociaciones con enfermedades específicas, como las lesiones cerebrovasculares y riesgos cerebrovasculares, debido a que en varios casos la presencia de síntomas depresivos es común después de un derrame cerebral que produce deterioro cognitivo (16).

\section{¿Cuáles funciones cognitivas se ven afectadas en presencia de depresión y DCL?}

En presencia de las dos patologías, se ha encontrado que en adultos mayores que poseen depresión y DCL diversas funciones cognitivas se ven afectadas. La evidencia señala que las funciones ejecutivas son las principales afectadas (25). Además existe bajo desempeño en tareas que implican memoria semántica, memoria episódica inmediata, memoria a largo plazo (26). Otros estudios sugieren que existen déficits cognitivos en funciones que están mayormente implicadas en el aprendizaje como atención y memoria, como también habilidades visuoespaciales (27). Se ha establecido que las mejores pruebas cognitivas para discriminar a los sujetos con depresión son la prueba de Dígitos (28) y el Test de Ejecución Continúa (CPT) (29), el Trail Making Test A and B (TMT-A) (30), el Test de Dígitos y Símbolos (28), el Test de Stroop (31), la pruebas de fluencia verbal y las pruebas de memoria inmediata (32).

Varios estudios se han enfocado en determinar si los síntomas de depresión se expresan de manera diferencial en subtipos de DCL. Un estudio (33) reportó que en individuos con DCL amnésico (DCLa), donde la función cognitiva más afectada es la memoria, síntomas de apatía eran más frecuentes, mientras que los individuos con DCL no amnésico (DCLna) presentaban problemas en los patrones de sueño. Otro estudio (34) reportó que personas con DCLa tenían mayor prevalencia en síntomas de depresión que los sujetos con DCLna, con una prevalencia de depresión de $83 \%$ y $73.1 \%$ en el grupo DCLa y DCLna respectivamente. Estos resultados sugieren que a simple vista diferentes síntomas de depresión se expresan en DCLa y DCLna, pero se requiere mayor evidencia para confirmarlo.

\section{¿Cómo se evalúa y diagnostica la relación DCL-depresión?}

La evaluación de la depresión en el DCL es multidimensional. Se realizan entrevistas psiquiátricas y psicológicas, exámenes médicos, exámenes de laboratorio y se evalúa si la persona cumple con los criterios de instrumentos diagnósticos como DSM-V o CIE-11 para depresión (35). Inicialmente es el cuidador o acompañante quién nota cambios en la conducta y cognición de la persona y solicita una cita médica. Los profesionales de la salud que evalúan al adulto mayor (geriatra, neurólogo, médico general) y sospechan de una depresión refieren al individuo a psiquiatría y/o psicología para confirmar la depresión o descartar cualquier otra condición. Una vez se inicia la evaluación se recomienda consultar primero con el cuidador, ya que la persona podría presentar déficits en funciones cognitivas como la memoria a corto plazo, el pensamiento abstracto o funciones 
Invest. pens. crit. (ISSN 1812-3864)

Vol. 6, No. 2, mayo - agosto 2018

pp. 05-12

ejecutivas, dificultando la capacidad de expresar o reportar humor deprimido (36). En ciertos casos, la información que da el cuidador puede estar afectada por cambios que el cuidador está atravesando como estrés u otros síntomas asociados al síndrome de degaste del cuidador (37). La entrevista inicial se realiza para armar una historia clínica del individuo y debe incluir información de los datos sociodemográficos, antecedentes personales (abarcando las distintas áreas: de salud, social, ocupacional, datos de relevancia de la niñez y adolescencia) y antecedentes familiares.

Existen diversos cuestionarios para medir los síntomas depresivos en el adulto mayor como el Geriatric Depression Scale, GDS (38), The Cornell Scale (39); The Montgomery Asberg Depression Rating Scale (MADRS) (40); The Hamilton Depression Rating Scale (41); The Hospital Anxiety and Depression Scale (42); Goldberg Depression Scale (43); Inventario Neuropsiquiátrico de Cummings (44). La evaluación de la depresión culmina con las recomendaciones pertinentes acerca de que tratamientos seguir para tratar el cuadro depresivo. Se comienza un tratamiento farmacológico y se establece la necesidad de terapia psicológica y/o estimulación o rehabilitación cognitiva para abordar los déficits cognitivos (45).

\section{Conclusiones}

La depresión es un trastorno mental que tiene complicaciones a todas las edades, sin embargo, en la edad adulta y en comorbilidad con DCL, es causante de mayor discapacidad y del desarrollo de demencias como de tipo EA. Es evidente la coocurrencia de la depresión y el deterioro cognitivo leve en la vejez, condiciones estrechamente asociadas a la edad avanzada. Múltiples estudios muestran que su origen puede estar relacionado con causas biológicas y/o factores psicosociales. La probabilidad de que la coocurrencia de la depresión y DCL avance a una demencia de tipo EA dependerá de la evaluación temprana e intervención oportuna.

\section{Referencias}

1. Naciones Unidas. La situación demográfica en el mundo 2014. A: . 2014,

2. Humbert, F.A. et al. Envejecimiento Demográfico en Panamá Periodo 1960-2050. A: . 2015.

3. World Bank. Expectativa de vida en Panamá. A: [en línia]. 2018. Disponible a:

http://www.worldbank.org.

4. World Health Organization. Mental health of older adults. A: . 2017.

5. Alzheimer's Association. 2017 Alzheimer's disease facts and figures. A: Alsheimer's Dement. 2017, p. 13:325-373.

6. Snowden, M.B. et al. Longitudinal association of dementia and depression. A: American Journal of Geriatric Psychiatry [en línia]. Elsevier Ltd, 2015, Vol. 23, núm. 9, p. 897-905. ISSN 15457214. DOI 10.1016/j.jagp.2014.09.002. Disponible a: http://dx.doi.org/10.1016/j.jagp.2014.09.002.

7. American Psychiatric Association. Guía de Consulta de los Criterios de Diagnóstico del DSM-V. 2013. 
Invest. pens. crit. (ISSN 1812-3864)

Vol. 6, No. 2, mayo - agosto 2018

pp. $05-12$

ISBN 9780890425510.

8. Aziz, R. i Steffens, D.C. What are the causes of late-life depression? A: Psychiatric Clinics of North America. 2013, Vol. 36, núm. 4, p. 497-516. ISSN 0193953X. DOI 10.1016/j.psc.2013.08.001.

9. Mitchell, A.J. i Izquierdo de Santiago, A. Prognosis of depression in the elderly in comparison with adult age. Is there a significant clinical difference? A: Actas españolas de psiquiatría [en línia]. 2009, Vol. 37, núm. 5, p. 289-96. ISSN 1139-9287. Disponible a:

http://www.ncbi.nlm.nih.gov/pubmed/19960388.

10. Mccall, W.V. i Kintziger, K.W. Late life depression a global problem with few resources. A: Psychiatric Clinics of NA [en línia]. Elsevier Inc, 2016, Vol. 36, núm. 4, p. 475-481. ISSN 0193-953X. DOI 10.1016/j.psc.2013.07.001. Disponible a: http://dx.doi.org/10.1016/j.psc.2013.07.001.

11. Ortiz, G.G. et al. Prevalence of cognitive impairment and depression among a population aged over 60 years in the metropolitan area of Guadalajara, Mexico. A: Current Gerontology and Geriatric Research. 2012, Vol. 2012, p. 1-7. DOI 10.1155/2012/175019.

12. Steunenberg, B. et al. Personality predicts recurrence of late-life depression. A: Journal of Affective Disorders [en línia]. 2010, Vol. 123, núm. 1-3, p. 164-172. ISSN 0165-0327. DOI

https://doi.org/10.1016/j.jad.2009.08.002. Disponible a:

http://www.sciencedirect.com/science/article/pii/S0165032709003504.

13. Steunenberg, B. et al. Personality and the onset of depression in late life. A: Journal of Affective Disorders [en línia]. 2006, Vol. 92, núm. 2-3, p. 243-251. ISSN 0165-0327. DOI https://doi.org/10.1016/j.jad.2006.02.003. Disponible a: http://www.sciencedirect.com/science/article/pii/S0165032706000784.

14. Blazer, D.G. Depression and social support in late life: A clear but not obvious relationship. A: Aging \& Mental Health [en línia]. 2005, Vol. 9, núm. 6, p. 497-499. DOI 10.1080/13607860500294266. Disponible a: http://dx.doi.org/10.1080/13607860500294266.

15. Bruce, M.L. Psychosocial risk factors for depressive disorders in late life. A: Biological Psychiatry [en línia]. 2002, Vol. 52, núm. 3, p. 175-184. ISSN 0006-3223. DOI https://doi.org/10.1016/S00063223(02)01410-5. Disponible a: http://www.sciencedirect.com/science/article/pii/S0006322302014105.

16. Alexopoulos, G.S. et al. «Vascular depression» Hypothesis. A: Archives of General Psychiatry [en línia]. 1997, Vol. 54, núm. 10, p. 915-922. DOI 10.1001/archpsyc.1997.01830220033006. Disponible a: + .

17. Bennett, S. i Thomas, A.J. Depression and dementia: Cause, consequence or coincidence? A: Maturitas [en línia]. Elsevier Ireland Ltd, 2014, Vol. 79, núm. 2, p. 184-190. ISSN 18734111. DOI 10.1016/j.maturitas.2014.05.009. Disponible a: http://dx.doi.org/10.1016/j.maturitas.2014.05.009.

18. Sampath, D., Sathyanesan, M. i Newton, S.S. Cognitive dysfunction in major depression and Alzheimer's disease is associated with hippocampal-prefrontal cortex dysconnectivity. A: Neuropsychiatric Disease and Treatment. 2017, Vol. 13, p. 1509-1519. ISSN 11782021. DOI 10.2147/NDT.S136122.

19. Kim, J.-M. et al. Synergistic associations of depression and apolipoprotein E genotype with incidence of dementia. A: International journal of geriatric psychiatry. England: 2011, Vol. 26, núm. 9, p. 893-898. ISSN 1099-1166 (Electronic). DOI 10.1002/gps.2621. 
Invest. pens. crit. (ISSN 1812-3864)

Vol. 6, No. 2, mayo - agosto 2018

pp. 05-12

20. Den Heijer, T. et al. A study of the bidirectional association between hippocampal volume on magnetic resonance imaging and depression in the elderly. A: Biological psychiatry. United States: 2011, Vol. 70, núm. 2, p. 191-197. ISSN 1873-2402 (Electronic). DOI 10.1016/j.biopsych.2011.04.014.

21. Panza, F. et al. Temporal relationship between depressive symptoms and cognitive impairment: The italian longitudinal study on aging. A: Journal of Alzheimer's Disease. 2009, Vol. 17, núm. 4, p. 899911. ISSN 13872877. DOI 10.3233/JAD-2009-1111.

22. Villarreal, A.E. et al. Cognitive impairment, depression, and cooccurrence of both among the elderly in panama: differential associations with multimorbidity and functional limitations. A: BioMed Research International. 2016, Vol. 2016, p. 7 pages. DOI 10.1155/2015/718701.

23. Lee, J.S. et al. Persistent mild cognitive impairment in geriatric depression. A: International psychogeriatrics. England: 2007, Vol. 19, núm. 1, p. 125-135. ISSN 1041-6102 (Print). DOI 10.1017/S1041610206003607.

24. Panza, F. et al. Late-life depression, mild cognitive impairment, and dementia: possible continuum? A: The American Journal of Geriatric Psychiatry [en línia]. American Association for Geriatric Psychiatry, 2010, Vol. 18, núm. 2, p. 98-116. ISSN 10647481. DOI 10.1097/JGP.0b013e3181b0fa13. Disponible a: http://dx.doi.org/10.1097/JGP.0b013e3181b0fa13.

25. Liao, W. et al. The characteristic of cognitive dysfunction in remitted late life depression and amnestic mild cognitive impairment. A: Psychiatry Research [en línia]. Elsevier Ireland Ltd, 2017, Vol. 251, núm. February 2016, p. 168-175. ISSN 18727123. DOI 10.1016/j.psychres.2017.01.024. Disponible a: http://dx.doi.org/10.1016/j.psychres.2017.01.024.

26. Sheline, Y.I. et al. Cognitive function in late life depression: Relationships to depression severity, cerebrovascular risk factors and processing speed. A: Biological Psychiatry [en línia]. 2006, Vol. 60, núm. 1, p. 58-65. ISSN 0006-3223. DOI https://doi.org/10.1016/j.biopsych.2005.09.019. Disponible a: http://www.sciencedirect.com/science/article/pii/S000632230501320X.

27. Johnson, L.A. et al. Cognitive differences among depressed and non-depressed MCI participants : a project FRONTIER study. A: International Journal of Geriatric Psychiatry. 2013, p. 377382. DOI $10.1002 / \mathrm{gps} .3835$.

28. Wechsler, D. Wechsler Memory Scale IV. Barcelona: Pearson, 2013.

29. Meneres Sancho, S. et al. Tests de ejecución continua: Integrated Visual and Auditory Continuous Performance Test (IVA/CPT) y TDAH. Una revisión. A: Revista de Psicología Clínica con Niños y Adolescentes [en línia]. 2015, Vol. 2, núm. 2, p. 107-113. ISSN 2340-8340. DOI http://www.redalyc.org/html/4771/477147186003/. Disponible a: https:/ / dialnet.unirioja.es/servlet/articulo?codigo=5150424\&info=resumen\&idioma=ENG.

30. Reitan, R. Validity of the Trail Making Test as an Indicator of Organic Brain Damage. A: Southern Universities Press. 1958, Vol. 8, p. 271-276.

31. Scarpina, F. i Tagini, S. The stroop color and word test. A: Frontiers in Psychology. 2017, Vol. 8, núm. APR, p. 1-8. ISSN 16641078. DOI 10.3389/fpsyg.2017.00557.

32. Lim, J. et al. Sensitivity of cognitive tests in four cognitive domains in discriminating MDD patients from healthy controls: a meta-analysis. A: International psychogeriatrics. England: 2013, Vol. 25, núm. 9, p. 1543-1557. ISSN 1741-203X (Electronic). DOI 10.1017/S1041610213000689.

33. Ellison, J.M. et al. Beyond the «C» in MCI: noncognitive symptoms in amnestic and non- 
Invest. pens. crit. (ISSN 1812-3864)

Vol. 6, No. 2, mayo - agosto 2018

pp. $05-12$

amnestic mild cognitive impairment. A: CNS spectrums. 2008, Vol. 13, núm. 1, p. 66-72. ISSN 10928529. DOI 10.1017/S1092852900016175.

34. Rozzini, L. et al. Neuropsychiatric symptoms in amnestic and nonamnestic mild cognitive impairment. A: Dementia and Geriatric Cognitive Disorders. 2007, Vol. 25, núm. 1, p. 32-36. ISSN 14208008. DOI 10.1159/000111133.

35. Mayo Clinic. Depression (major depressive disorder). A: Mayo Clinic [en línia]. 2018. Disponible a: https://www.mayoclinic.org/diseases-conditions/depression/diagnosis-treatment/drc-20356013.

36. Pellegrino, L.D. et al. Depression in cognitive impairment. A: Curr Psychiatry Rep. 2013, Vol. 15, núm. 9, p. 384. ISSN 1535-1645. DOI 10.1007/s11920-013-0384-1.

37. Rosenberg, P.B., Mielke, M.M. i Lyketsos, C.G. Caregiver assessment of patients' depression in Alzheimer disease: Longitudinal analysis in a drug treatment study. A: The American Journal of Geriatric Psychiatry [en línia]. 2005, Vol. 13, núm. 9, p. 822-826. ISSN 1064-7481. DOI

https://doi.org/10.1097/00019442-200509000-00012. Disponible a: http://www.sciencedirect.com/science/article/pii/S1064748112609555.

38. Yesavage, J.A. et al. Development and validation of a geriatric depression screening scale: A preliminary report. A: Journal of Psychiatric Research [en línia]. 1982, Vol. 17, núm. 1, p. 37-49. ISSN 00223956. DOI 10.1016/0022-3956(82)90033-4. Disponible a: http:/ /www.ncbi.nlm.nih.gov/pubmed/7183759\%5Cnhttp://linkinghub.elsevier.com/retrieve/pii/ 0022395682900334.

39. Alexopoulos, G.S. et al. Cornell Scale for Depression in Dementia. A: Biological psychiatry. United States: 1988, Vol. 23, núm. 3, p. 271-284. ISSN 0006-3223 (Print).

40. Montgomery, S.A. i Asberg, M. A new depression scale designed to be sensitive to change. A: The British journal of psychiatry : the journal of mental science. England: 1979, Vol. 134, p. 382-389. ISSN 0007-1250 (Print).

41. Hamilton, M. A rating scale for depression. A: Journal of neurology, neurosurgery, and psycbiatry. England: 1960, Vol. 23, p. 56-62. ISSN 0022-3050 (Print).

42. Zigmond, A.S. i Snaith, R.P. The hospital anxiety and depression scale. A: Acta psychiatrica Scandinavica. United States: 1983, Vol. 67, núm. 6, p. 361-370. ISSN 0001-690X (Print).

43. Goldberg, D. et al. Detecting anxiety and depression in general medical settings. A: BMJ (Clinical research ed.). England: 1988, Vol. 297, núm. 6653, p. 897-899. ISSN 0959-8138 (Print).

44. Cummings, J.L. et al. The Neuropsychiatric Inventory: comprehensive assessment of psychopathology in dementia. A: Neurology. United States: 1994, Vol. 44, núm. 12, p. 2308-2314. ISSN 0028-3878 (Print).

45. Morimoto, S.S. et al. Diagnosis and Treatment of Depression and Cognitive Impairment in LateLife. A: Ann N Y Acad Sci. 2015, Vol. 1345, núm. 1, p. 36-46. DOI 10.1111/nyas.12669.Diagnosis. 